\title{
No Fault Found Events in Maintenance Engineering Part 2: Root Causes, Technical Developments and Future Research
}

\author{
Samir Khan ${ }^{\mathrm{a}, *}$, Paul Phillips ${ }^{\mathrm{a}, *}$, Chris Hockley ${ }^{\mathrm{b}, *}$, Ian Jennions ${ }^{\mathrm{c}, *}$ \\ ${ }^{a}$ EPSRC Centre, School of Applied Sciences, Cranfield University, College Road, Cranfield, Bedfordshire, MK43 OAL \\ ${ }^{b}$ Cranfield Defence and Security, Cranfield University The Mall, Shrivenham, Oxfordshire, SN6 8LA \\ ${ }^{c}$ IVHM Centre, School of Applied Sciences, Cranfield University, University Way, Cranfield, Bedfordshire, MK43 OFQ
}

\begin{abstract}
This is the second half of a two paper series cover aspects of the NFF phenomenon, which is highly challenging and is becoming even more important due to increasing complexity and criticality of technical systems. Part 1 introduced the fundamental concept of unknown failures from an organizational, behavioral and cultural stand point. It also reported an industrial outlook to the problem, recent procedural standards, whilst discussing the financial implications and safety concerns. In this issue, the authors examine the technical aspects, reviewing the common causes of NFF failures in electronic, software and mechanical systems. This is followed by a survey on technological techniques actively being used to reduce the consequence of such instances. After discussing improvements in testability, the article identifies gaps in literature and points out the core areas that should be focused in the future. Special attention is paid to the recent trends on knowledge sharing and troubleshooting tools; with potential research on technical diagnosis being enumerated.
\end{abstract}

Keywords: No fault found; test equipment; troubleshooting failures; fault diagnostics; maintainability; testability

\section{Introduction}

Part 1 extensively discussed the organizational complexities and challenges faced by businesses today in attempts to administer solutions to the problems caused by unidentified failures. It also described the applied method for collection and analysis of the referenced literature in detail. This was included not only to judge the validity of these papers, but also to present a statistical analysis of the academic journal publications on NFF concepts between the period 1990-2013. In addition, the authors had categorized the literature into four main areas: fault diagnostics, system design, human factors and data management, where it was noted that fault diagnostics and system design have been the main focus for NFF journal publications within the past two decades. Part 1 also focused on No Fault Found (NFF) standards, and how such events can cause unprecedented changes in the service performance, impact dependability and escalate safety concerns. This has long been revealed with a variety of products, within a wide range of industries [1, 2, 3, 4].

This paper aims to elaborate on these outlooks (from Part 1), whilst examining the technical aspects for complex systems and equipment (particularly products integrated within aircraft computer systems), and how such events can have a significant effect upon the overall unit removal rate. Historically, such removals have been seen as an unavoidable nuisance [5], but this viewpoint is no longer acceptable if the unit removal rate is to be managed effectively $[6,7]$. Unlike those failures that result in 'Confirmed Faulty' events, the designer may have no

\footnotetext{
* Corresponding author. Tel: +44 (0)1234 750111
}

E-mail address: samir.khan@ @heiet.org direct influence on those aspects of the system that determine the NFF failure rate, therefore a direct mitigating action during the design phase is likely to be more difficult ${ }^{1}$. It can be argued that any product removal that does not exhibit a failure (during subsequent acceptance test) can be tagged as NFF. Also, for a number of these events, further investigation could conclude that the reason for the removal event was categorically caused by an external effect. None-the-less, this would still be classified as a NFF event as these external influences might be faulty sensors (or actuator), or possibly an incorrect fault isolation activity. In any case, as the device fabrication process continues to improve, failure rates of hardware components have steadily declined over the years to the point where non-hardware failures emerged as a dominant issue [9]; where as the reduction of troubleshooting complexities and time to fix a problem seem to be the most important aspects when investigating failures of electronic systems.

In addition to the a priori discussions from Part I, this paper focuses on the following:

1. No Fault Found Occurrences in Systems

2. Emerging Resolution Practices

3. Improvements in Test Abilities

4. Discussion on Gaps in Literature

5. Future Research Directions

\footnotetext{
${ }^{1}$ Although, there are specific approaches, such as 'robust design' [8], that can be used to design quality into products and processes; by minimizing the effects of the causes of variation, without eliminating the cause.
} 
The remainder of the paper is structured as follows; after identifying the common root causes for NFF in system components, the brief survey's some industry specific innovations that have been introduced in order to capture troubleshooting data. Section 4 discusses improvements in test capabilities; followed by a discussion on the identified gaps in NFF literature. Finally concluding remarks and future directions for research into testability methods, and the necessary design guidance to mitigate the problem are cover in Section 6.

\section{No Fault Found Occurrences in Systems}

\subsection{Electronic Systems}

Electronic failures are not often considered as static nor random (or pseudorandom) events, but rather the result of mechanical and material changes $[9,10]$. These changes seldom lead to a loss of functionality of an electronic system, even though their components maybe out of specification. This is due to the electronics having an inherent self-compensating aspect that makes the task of failure diagnostics difficult and directly contributes to a successful diagnosis. In addition, degradation of failure modes often manifest differently depending upon the operating environment that may offset components and the circuit configuration [11]. Thomas et al. (2002) [12] and Renner (1999) [13] investigated the root causes of NFF in automotive electronic systems. It was revealed that an overwhelming majority of occurrences can be traced back to poor manufacturing (i.e. soldering and Printed Circuit Board (PCB) assembly) and inherent design flaws which include violation against specifications. Vichare and Pecht (2006) [10], Qi et al. (2008) [14] and Moffat (2008) [15] have summarized some generic causes of failures within electronic systems:

\section{Interconnect failures (including connectors)}

2. System design (electrical and mechanical)

3. Environmental conditions (temperature, moisture, chemicals, mechanical stresses)

4. Operator handling (ergonomics, training)

5. Printed circuit Boards (PCB)

6. Ageing components and connectors

7. Loose PCB interconnectors

8. Disconnected solder points

9. Damaged wiring or cabling

A recent aerospace survey [16] has ranked intermittent faults as the major cause of NFF events, whereas Built-In-Test Equipment (BITE) coverage and software are least likely. This is contrary to the common belief that the majority of failures are due to incompatible or competing software routines between systems [17]. Intermittency is arguably the most problematic of the NFF events due to their elusive nature, making detection by standard test equipment difficult [5]. The faulty state will often lay dormant until a component is back in operational use, where it eventually causes further unit removals unless a genuine cause is found (fault isolation). It should be emphasized that these failures are not always present during testing, which make them troublesome to isolate. This situation can result in repeated removals of the same equipment for the same symptom, with each rejection resulting in the equipment being tagged as NFF [18]. At this stage, there is a very high probability that there will be a loss of system functionality, integrity and an unacceptable compromise in safety requirements. What is clear is that even though these faults may begin as short duration low frequency occurrences, as time passes the underlying cause will increase the severity of the intermittency until eventually a hard fault appears and the functionality of the system is compromised or lost.

\subsubsection{Printed Circuit Board Interconnectors}

Information published by Gibson et al. (1997) [19], claims that between $50-70 \%$ of all electronic device failures could be attributed to its interconnectors. Even though solder joints can fail by a variety of mechanisms, the device 'interface' seems to be the most common cause ${ }^{2}$. Over time, contaminations on the fractured surfaces initiate a failure sequence which starts with degraded joints and eventually progress to intermittent failures.

Products that have a dependency upon the behavior of interfacing devices for correct operation are also susceptible to faults which can be categorized as intermittent. This is common in products that rely on software for their correct operation (or interaction) with other products. In these cases, they may exhibit periodic failures due to inherent incompatibilities between the system interfaces; symptoms may include relative timing errors and synchronization issues. The systems may not show any evidence of failure for many years of service, but as the system interfaces become affected by wear and drift, failures become evident. This can result in a root cause misclassification, with the root cause being diagnosed as component ageing rather than the fundamental design issue with the interface.

Another major contributor to solder joint damage is thermal stress related to heat expansion, shock and vibration. During operation, these stresses causes metal-metal interconnects to rub against each other to damage any protective coating. Such effects cumulate over time, and will typically last for periods less then hundreds of nanoseconds. Such manifestations fracture the solder contacts and instigate intermittent faults. Electrical intermittency is also caused by contact fretting $[15,20]$. Fretting corrosion occur particularly in tin plated contacts, as a degradation mechanism caused by the presence of humidity which oxidizes the metal-metal interface. The accumulation of oxides at the contacts causes an increase in resistance and electrical intermittency due to the repetitive sliding movements.

Other root causes of NFF events in electronics include creep corrosion, and the phenomena known as tin whiskers [14]. Creep corrosion is a mass transport process in which solid corrosion products migrate over a surface on Integrated Circuit (IC) packages and eventually result in electrical shorts (or signal deterioration) due to the bridging of corrosion products between isolated leads. Depending on the nature of corrosion

\footnotetext{
${ }^{2}$ These failures can occur under several scenarios, a common failure is where surface-mount packaging used are knocked off during socket insertion.
} 
product (conductive or semi-conductive, dry or wet), the insulation resistance can vary, thus potentially causing intermittent loss of signal integrity. A pure tin finish is well known to produce conductive 'metal whiskers', that are capable of producing unintended current paths. These failures usually appear intermittently, making it difficult to identify them as a root cause to the problem; they are easily broken off and can melt to remove a previously existing $\operatorname{short}^{3}$ [8]. In the case of a reported failure where there is no 'hard' (or definite) symptom for a sufficient fault diagnosis; there will be the need for additional technical data or specialist technical knowledge. This can be in the form of maintenance history, troubleshooting guides or expertise from experienced colleagues and specialists [2, 5].

\subsubsection{Harness Wiring}

A key aspect of interconnect and wiring related failures is that they will often not be detected by traditional one-path-at-atime sequential mode of analysis [22]. The traditional approach not only fails to spot time-dependant failures (such as those exhibited under vibration), but could inherently ignore combinatorial faults that occur due to wire-to-wire interactions. Another issue is when chafed wiring occurs where a harness is routed through a structure that experiences high vibration levels. Unless adequate protection (such as cable clamps, ties, sleeving etc) are provided, the wiring bundle will brush the structure in such a way that damages internal wiring without external evidence. Such type of wiring faults are extremely difficult to detect and can lead to risk the maintenance crew rejecting products incorrectly, which are associated with this particular signal path. Wire breaks are common in harnesses, and are likely to manifest as a hard fault for a period determined by the vibration and temperature profile. However, in order to correctly isolate the failure in an ambient environment, stressing of the harness may be necessary to simulate the conditions in which the failure occurred. In cases where fault is intermittent and the exact operating conditions are not known, the failure may not be correctly attributed as 'being in the harness'; which will lead to the suspicion that the unit is at fault and requires replacing. This is particularly true for those maintainers who operate within the constraints of fast turnaround times.

\subsection{Mechanical Systems}

The failure mechanisms within a mechanical system are widely regarded as having less of an effect upon the rate of NFF occurrences than those which are present within electrical systems. The causes of failure in mechanical systems are similar to those in electrical systems, such as ageing, poor maintenance, incorrect installation or usage. The difference however is that it is much easier to predict the effect upon the systems operation with mechanical failures. As a result this allows inspection criterions to be developed during the design phases [23]. It should be noted that as with many electrical failures, mechanical failures can be intermittent in nature and only occurring under spe-

\footnotetext{
${ }^{3}$-also, tin whisker growth is much more likely in lead-free solder to cause short circuits [21].
}

cific operating conditions. Some of the more common mechanical failures which are of interest but receive a lot less attention then the electrical failures which contribute to diagnostic failure are:

1. Broken seals and leaks: Leaks from broken seals will affect the operation of items which include engines, gearboxes, control actuators and hydraulic systems. The nature of seal design is that they are often designed to slightly weep. This is a good example of the need for maintenance personnel to be familiar with the system and hence be aware of what constitutes acceptable leakage in order to avoid unnecessary removals.

2. Degradation of pneumatic and hydraulic pipes: Degradation within pipes often occurs due to corrosion or fretting against other components or structures. The nature of pneumatic/hydraulic systems is that under pressure they may develop small leaks. These minor leaks may result in an alarm to the operator indicating failure, resulting in the unwarranted shut down of the system, when no equipment malfunction has actually occurred.

3. Backlash in mechanical systems: One area where backlash can cause significant concern is within actuation systems, particularly those used for aircraft control surfaces. It is possible that with excessive wear in actuator couplings, position sensors may indicate incorrect operation, including asymmetric settings, which are difficult to isolate from a maintenance perspective.

\subsection{Software Systems}

It is clear that a great deal of NFF occur in avionics, electrical and electro-mechanical systems, however research discussions have also revealed that software (including Built-In-Tests (BIT)) is also a key contributor to the problem [5, 24, 25, 26]. This includes:

\section{Processing delays}

2. Discrepancies between software testing procedures

3. Timing errors

4. Lack of appropriate training

5. Perhaps a poorly written program code

Industry specific standards exist (such as IEC 62278 [27] for railways, or the IEC 60812 [28] is often referred to when carrying out Failure Mode and Effects Analysis (FMEA) ${ }^{4}$ for software based systems), that can be used to validate software operation and meet specific requirements. However, since standards and guidelines are prepared to be generic, they only briefly consider the handling of any malfunctions caused by

${ }^{4}$ FMEA (Failure Mode and Effects Analysis) is recognized as one of the most effective methods to identify and remove critical reliability issues. This procedure is commonly used to influence the system design before it is commissioned, enumerating potential failure modes that may occur during operation. These are proactively performed to assess the impact of various failure modes during the product development and maintenance stages [14]. Risk priority numbers can also be assigned to each of the failure modes, based on factors such as detectability, severity, and occurrence. 
software faults and their effects in FMEA [29]. Software components are often delivered with little access to the source code, which only provides a partial view of their internal functionality. With restricted access in these Off the Self (OTS) solutions, unpredictable effects and integration faults are likely to undermine critical software functions, which can be difficult to diagnose and locate [30]. Investigations into failures within aerospace missions have highlighted critical failures that are due to such components, along with incomplete software specifications [31]. Many of the reported issues in this paper can be attributed to complacency and misunderstanding of software functions, in the way they interact, and the lack of applying good practice principles.

In many cases, desired sources of information are not readily available, or are incorrectly configured to support rapid diagnostics, or lack sufficient depth of information and practicality. Additional factors include the failure to complete (or store) documentation and the lack of robust diagnostic fault trees connecting event-system-faults [5]. This results when a unit is replaced without determining the nature of the fault, risking its recurrence to cause an NFF event. The complexity brought by embedded software and electronics poses unprecedented challenges in maintenance and repair, threatening customer satisfaction and causing increasing warranty cost on repair [32, 33].

\section{Emerging Resolution Practices}

From a technical standpoint, an NFF tagged component is the result of an unsuccessful (or inefficient) troubleshooting regime of an 'unplanned maintenance event'. Several maintenance strategies are usually sought to improve upon this problem within organizations:

1. Reliability: If all components were $100 \%$ reliable (i.e. they never resulted in a system failure) then there would be no unplanned maintenance activities. Design engineers often engage in reliability improvements based largely on feedback from equipment in service. However, to the extent that engineers anticipate failures, designers will incorporate fault detection systems, notably BIT and prognostic strategies to keep track.

2. BIT: If BIT's were $100 \%$ comprehensive and unambiguous at the aircraft level (including interacting systems [34]) then it would:

i Detect every possible problem

ii Point with certainty to the defective part, and only where the problem was caused by a defective part (as opposed to operator mishandling, environmental circumstances, etc).

But, to the extent that BIT is lacking, troubleshooting is required.

3. Troubleshooting: In theory, if Fault Isolation Manuals (FIM), or troubleshooting guides, were perfect, then every failure that can occur on any aircraft would be swiftly (and correctly) identified by any maintenance personnel, following step-by-step procedures. However, FIM fails to identify the problem; the maintainers rely heavily on their experience [5]. Other resources are often used to help escalation channels, technician training, supporting documentation, etc.

4. On-site or practical feedback: To close the loop with reliability, new system failure modes are often discovered adding to the troubleshooting difficulties [26], and acts as a source of feedback to design engineering for reliability improvements.

\subsection{Health and Usage Monitoring}

Condition Based Maintenance (CBM) programmes can be aimed at either fault diagnostics or prognostics ${ }^{5}$ [35]. Diagnostics refers to a posterior event analysis and deals with fault detection (indicates a fault has occurred), fault isolation (faulty component is identified) and fault identification (the nature of the fault is determined). Prognosis is a prior event analysis and deals with failure prediction before faults occur, making use of in-situ sensors and physics-of-failure models [27]. If it is possible to assess in-situ the extent of degradation of electronic systems, then such data would be invaluable in meeting the objective of providing efficient fault detection and identification. This would include evidence of 'failed' equipment found to function correctly when tagged (as NFF) and hence improve maintenance processes, extend life, reduce whole life costs and improve future designs.

There is currently a drive in the majority of industries to turn away from the more traditional preventive and reactive maintenance actions described above in favor of more predictive and proactive solutions [21]. Condition Based Maintenance (CBM) is often regarded as the most advanced predictive maintenance strategy and hence, could be aimed at reducing the number of machinery breakdowns by fault detection at an early incipient stage [5, 10, 36]. CBM makes use of measurements of physical parameters while monitoring the trends over time; any indication of abnormal behavior will trigger a warning. In its simplest form, threshold warning levels are constructed to trigger maintenance activities when a specific parameter shows measurements outside of the threshold regions. In corrective maintenance, much of the time is spent on locating a defect which often requires a sequence of disassembly and reassembly. Recently, condition monitoring of railway wheels with NFF problems was investigated by Granstrom and and Soderholm (2009) [37]. The authors provided a perspective on how such technologies can be applied and utilized for more effective and efficient maintenance management, while initiating a discussion on the maintenance requirements of systems and the management regimes which are forced onto those systems. The ability to automate fault diagnosis, with advanced technologies and techniques, could be used to accurately predict the downtime and hence the operational availability. In fact, the role of diagnosability analysis in modern systems, considering their complexities and functional interdependencies, becomes significant as

5 - there are other maintenance programmes that do not consider diagnostics or prognostics, e.g. in time-based preventive maintenance where replacement of parts is performance after a predetermined time interval (measured by a relevant time measure, e.g. hours, cycles or tonnages), independent of the condition. 
it improvements can lead to a reduction of a system's life-cycle costs [38]. However, it should be noted that such setups are only worthwhile if the benefits can significantly outweigh the costs of its introduction and upkeep. There are design constraints often involved with improving maintainability, particularly in the airline industry when dealing with legacy aircraft. The more general issues include [39]:

1. Any technological enhancements must work within existing architectures

2. The information available from lower test levels are typically predefined and costly to improve or change.

3. Hardware development can be costly and outweigh potential cost saving benefits.

4. There may be limited space for additional processing capabilities to support improved diagnostics.

However, the authors would like to emphasize that if there are no safety (or operational) related consequence of the failure, then corrective maintenance is probably the most effective maintenance approach to be adopted. The choice of an appropriate strategy for the failure management is guided by methodologies such as 'Reliability Centered Maintenance (RCM) ${ }^{6}$, $[42,43]$ for military aviation and other applications, or 'Maintenance Steering Group-3 (MSG-3) ${ }^{7}$, [46] for civil aviation.

\subsubsection{Monitoring and Reasoning of Failure Precursors and Loads}

The basis of health monitoring is built upon the premise that there exist precursor indications of failure in the form of some change in a measurable parameter/signal of the system which can be correlated with a subsequent failure mode [9, 47]. Using this causal relationship, it is assumed that failures can then be predicted with the correct approaches to reasoning. The first step in health monitoring is to select the life-cycle parameters to be monitored. This can be done systematically through a Failure Mode Event and Criticality Analysis (FMECA) ${ }^{8}$. For example, a measurable parameter which can provide an indication of impending failure (or a 'failure precursor') for cables and connectors can include impendence changes, physical damage or a high-energy dielectric breakdown. By monitoring changes in these precursors, a system's health status and additional prognostic information can be evaluated, and unexpected failures could be avoided. A summary of potential failure precursors for electronics is defined by Born and Boenning (1989) [49].

The life-cycle environment of a product consists of manufacturing, storage, handling, operating and non-operating conditions, which may lead to physical/performance degradation

\footnotetext{
${ }^{6}$ Reliability Centered Maintenance (RCM) is a structured approach to ensure that assets continue to do what their users require in their present operating context $[40,41]$.

${ }^{7}$ Maintenance Steering Group-3 (MSG-3) based maintenance provides a top-down approach to determine the most applicable maintenance schedule, and the interval for an aircraft's major components and structure. The methodology effectively delivers significant improvements in an aircraft's availability and operational safety, whilst optimizing the costs of ownership [44, 45].

${ }^{8}$ Failure mode effects and criticality analysis (FMECA) is an extension of FMEA [48].
}

of the product to reduce its service life. Suppliers and operators, particularly within the airline industry, spend significant resources attempting to determine the root causes of the NFF events, but without any measured field conditions, a root cause analysis can be problematic for capturing information. This poses an even more significant challenge that requires additional specific sensing equipment and data loggers. Burns et al. (2002) [50] demonstrate the development, laboratory and in-flight testing of such specific equipment for monitoring the environment of aircraft avionic power system. The equipment termed the 'Aircraft Environment Monitor Power Quality (AEM PQ)', allows over two years of continuous data measurements to be collected for evaluation of the quality of power systems for different operational scenarios. The hardware and data gathered is a prime example of the information gathering abilities which are required to evaluate the influence of life-cycle loads on a specific mission critical system. The added bonus of this data is that it provides the foundations to troubleshooting NFF's, which can aid in re-evaluating system (avionic) design and establishing models for life cycle analysis.

Life cycle monitoring has been used to conduct prognostic Remaining Useful Life (RUL) estimates of circuit-cards inside of a space shuttle's solid rocket booster [51]. Vibration time history was recorded throughout all stages of the shuttle's mission and used with physics-based damage assessment models to predict the health and time before the next expected electronic failure. A similar methodology was applied to the end effector electronics unit inside the space shuttle's remote manipulator systems robotic arm [52]. In this case, loading profiles for both thermal and vibrational loads were used with damage models, inspections and accelerated testing to predict the component integrity over a 20 year period. Lall et al. (2007) [53] presented a methodology to calculate prior damage in electronic interconnects operating in harsh environments and hence subjected to highly cyclic and isothermal thermo-mechanical loads with assessment predictions in good correlation with experimental data using a health monitoring tools.

Understanding electronics from a system point of view, rather than a set of individual components, is claimed by VEXTEC Corporation to be paramount to developing life-cycle prognostic models as part of a failure reduction methodology [11]. The proposed methodology has far reaching consequences on how the operators can manage a fleet of aircraft based upon risk, rather than guessing degradation levels. It is argued that by doing this, NFF failure events can be reduced by the ability to prioritise the order of components replaced during a reported failure event, based on probabilities. Developing methodologies and damage assessment algorithms are generally aimed at creating an in-situ load monitoring and prognostic capability. This is explored by Vichare et al. (2007) [54] who provides the necessary considerations for raw data processing during in-situ monitoring and methods to reduce memory requirements and power consumption. These are key factors that often limit the integration of health monitoring systems, particularly into aircraft. Skormin et al. (2002) [55] developed failure prognostics for aircraft avionics using data mining models with measured parameters which included vibration, tempera- 
ture, power supply, functional overload and air pressure. These parameters, measured in-situ use time stress measurement devices. The purpose of the model included understanding how the role of measured environmental factors impact upon a particular failure, investigating the role of combined parameter effects and to re-evaluate the probability of failure on the known exposure to adverse conditions.

\subsubsection{Knowledge Sharing}

Engineers have recently empathized that there is need for 'on-field experience' to be shared within a troubleshooting workflow repository [21]. Aspects of content sharing (such as e-maintenance [56]) can be beneficial for other maintenance personnel who will then be able to identify the cause of a problem on their first attempt, whenever (or wherever) it next occurs. Furthermore, the captured knowledge, over time, can assist designers in improving the reliability of the equipment.

At the core of the challenge for better troubleshooting is the difference between 'anticipated failures' captured within the design and the 'actual failures' that appear in service. When complex equipment is designed, engineers typically identify the potential failure modes and their effects on the system using a FMEA. With this information, it can be determined how best to employ On-Board Diagnostic (or BIT) technologies to detect failures. These can implement Prognostics and Health Monitoring (PHM) strategies to detect impending functional failures. In addition, this can also prepare troubleshooting procedures, in advance, for analyzing the functionality of the system in order to differentiate among the many possible root causes of these anticipated failures. Procedures are contained in troubleshooting manuals or guides which require human involvement to execute the tests and evaluate the results. As good as they are, these systems are often far from perfect nor should they be expected to be, given the necessary practical cost/performance tradeoffs [5, 57]. Furthermore, existing RCM standards (such as IEC 60812 [29] FMEA, IEC 60300-3-11 [42], SAE JA1012 [43]), and experts related to FMEA (Moubray (1997) [41], Stamatis (1995) [58]), emphasize the importance of continuously updating them and making sure that it is a 'living' document that reflects new knowledge and gained experiences. This importance of continuous improvement is also emphasized by related standards such as IEC 60300-3-14 [53] and EN 50126 [27] (or IEC 62278 [52]). It should be highlighted that FMEA analysis directly contributes to the development of effective maintenance procedures (e.g. RCM and MSG-3 in the aircraft industry incorporate FMEA as the primary component of analysis), as well as the identification of troubleshooting activities, maintenance manual development and design of effective built-in-test requirements.

When the equipment enters service, the 'Practical World' imposes itself, as shown in Fig. 1, some faults that were anticipated will actually happen; but some never do. When a fraction of the theoretically possible failure modes occur, the weaknesses in a piece of equipment will become evident during the operation. It can then be extrapolated that equipment which fail on one aircraft, are more likely to fail on other aircraft of the same design, operated in similar conditions. But most im- portantly, many real-world faults are not anticipated by the design engineers, and therefore the traditional diagnostic systems do not resolve them. In those cases, human ingenuity may resolve the problem but where does that knowledge reside after its creation? Some the knowledge can make its way back into troubleshooting manual updates [36, 59], and some may be fed back to engineering to modified designs for much more reliable parts [60]. However, most of the knowledge only resides within the heads of a few key experts, or in personalized organizational databases which usually are consulted only after a problem has resisted several attempts at resolution. Therefore, on-site experience must be blended with other diagnostic and prognostic tools and techniques [42]. The obvious challenges here are:

1. To store this experience-based knowledge, and deliver it at the time and place that the same problem symptoms occur, so that it can be re-used to help solve the problem on the first attempt.

2. To deliver that knowledge in a form that is useful to experts and less-experienced technicians alike.

3. To share this knowledge so that everyone benefits from the experience of others

4. To integrate the knowledge access with the existing troubleshooting tools so that it becomes part of the usual troubleshooting workflow.

Human factors must be considered with respect to troubleshooting performance [61]. A diagnostic reasoning system could hence be useful to provide an such information, along with high quality feedback to the design engineers [62]. With the entry of symptoms, the possible failure modes can be identified from the knowledge database ${ }^{9}$, and increasingly incisive information can be requested. To the troubleshooter, this can act as efficient guidance; to the design engineer, this can be an intelligent interview automatically being applied anytime that these failures modes appear. When completing the troubleshooting, the maintainers can automatically report on the failure mode and record detailed differentiating symptoms. Also, this information can be of great importance for a 'Failure Reporting, Analysis, and Corrective Action System' (FRACAS) ${ }^{10}$, procedure, providing valuable insights to engineers [42, 64].

\subsection{Test Equipment}

Automatic Test Equipment (ATE) is widely used to perform device functional and parametric tests at the back-end of the semiconductor manufacturing process [9]. It is a capital intensive system and typically costs $\$ 1-\$ 3 \mathrm{M}$ depending on the equipment performance. An unscheduled equipment downtime lasting one hour could cause significant amounts of production loss.

\footnotetext{
${ }^{9}$ Various reliability and maintenance databases can been compiled, such as [63], eliciting information useful in scheduling maintenance and design activities.

${ }^{10}$ FRACAS (Failure Reporting, Analysis, and Corrective Action System) is a reactive procedure often utilized after failures have occurred within a system. It is used to collect data, report, categorize, analyze information, and to plan corrective actions in response to those failures.
} 


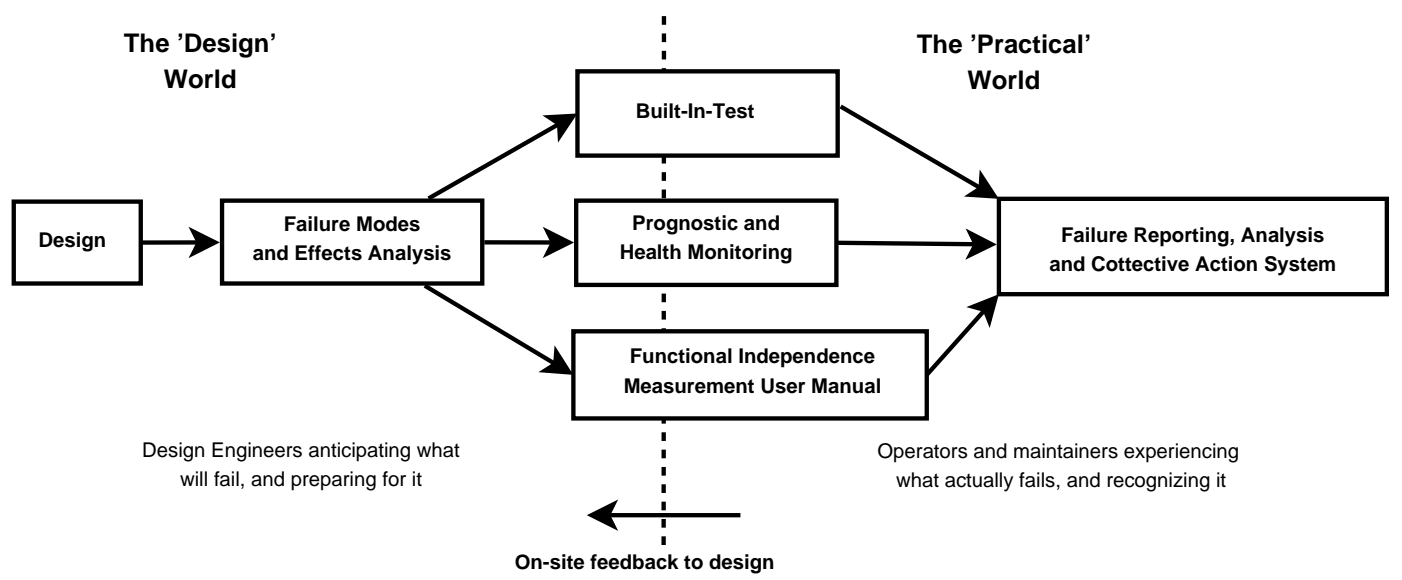

Figure 1: Troubleshooting: Anticipated vs Actual Faults.

The use of reflectometry has commonly been used to determine the integrity of cables and wiring with effective localization of intermittent faults such as open or short circuits. These methods send a high frequency signal down the line, which reflects back at impedance discontinuities. The location of the fault is determined by the phase shift between the incident and reflected signals. Sharma et al. (2007) [65] demonstrates a novel architecture for implementing a Sequence Time Domain Reflectometry (STDR) method, which uses a pseudonoise code to locate open and short circuits on active wires using an integrated CMOS sensor. The approach has an accuracy of fault localization of $1 \mathrm{ft}$ with low power consumption for the sensor. Lo and Furse (2005) [66] provide research into similar faults but using a differing kind of reflectrometry known as Noise-Domain Reflectrometry (NDR) which make use of existing data signals in the wiring. With this method results show the potential to localism intermittent faults within 3 inches in $180 \mathrm{ft}$ of electrical wiring. However, caution must be taken when using these methods as little is known on the impedance profile of intermittent faults (with exception to open and short circuits). Also promising are reflectometry methods, that are proving to be useful when applied to locating intermittency in an F-18 flight control harness [67]; they do require exceptional accuracy in baseline comparisons. In civil and military aerospace, recording and maintaining TDR data archives, for even a limited number of circuit's, may prove to be enormous and costly [68]. Another technique, called spread-spectrum time-domain reflectometry (SSTDR) is commercially being used to identify faults in electrical wires by observing reflected spread spectrum signals Parkey et al [69].

CMOS Integrated Circuits (IC) are routinely tested using supply current monitoring which is based upon the knowledge that a defective circuit will produce a significantly different amount of current than fault-free circuits. Smith and Campbell (2000) [70] have developed an in-situ quiescent current monitor that detects, in real-time, elevations in the leakage current drawn by the IC whilst in a stable state. Other similar current monitors have been reviewed by Pecht (2006) [43]. Damage to electronic solder joints are a major contributor to intermittency in electronics and hence are a direct contributor to the NFF phenomena. Damaged solder points are notoriously difficult to detect without extensive visual inspections. They do however produce large variations in thermal resistance which can be used as a potential suitable method for monitoring solder joint fatigue inside of the packaging of power modules. Bhatia et al. (2010) [71] have used this principle as a basis to develop and test a new solder-joint fault sensor known as the SJ Monitor which provides the ability to monitor selected $\mathrm{I} / \mathrm{O}$ pins of powered-off FPGA's. The use of RF impedance is also used as a failure precursor and offers interesting prognostic capabilities for solder joint failures due to the nature of gradual non-linear increases in impedance as damage increase, whereas the DC resistance becomes constant. The use of RF impedance is researched at length by Kwon (2010) [72], who demonstrates prognostic capabilities which are able to predict the remaining useful life of the solder joint with an error less than $3 \%$. The research also demonstrates the ability to distinguish between two competing interconnects failure modes solder joint cracking and pad cratering; the need for such failure distinctions in this case however is unclear.

The use of embedded molecular test equipment within ICs enabling them to continuously test themselves during normal operation, providing visual indications of failure has been proposed by GMA Industries as one of the more advanced and futuristic monitoring technologies [29]. The sensors are used to measure electrical parameters and various signals such as current and voltage, as well as sensing changes in the chemical structure of integrated circuits that are indicative of developing failure modes. The basic structure of the sensors are carbon nanotubes and the integration of these sensors with conventional IC's along with molecular wires for the interconnecting sensor networks is the important focus of this research. However no details of demonstrable in-service products or prototypes are given and to date no research paper offering proofs on the applicability of the concept has been found.

Recently, a sensitive analyzer was introduced by Universal Synaptic to simultaneously monitor test lines for voltage variation, and seems to have become an attractive tool for detec- 
tion of the intermittency $[73,74]$. Conducting the intermittency test simultaneously provides an increase in probability of detection; combined with the reduction in the time taken to complete the test (because the testing is performed for multiple points, rather than testing one line at a time) means that this is potentially an effective test methodology. It has been used on the F-16 AN/APG-68 Radar system Modular Low Power Radio Frequency (MLPRF) unit where \$36 million dollars' worth of assets, previously deemed 'unrepairable' have been returned as serviceable. The equipment has also shown considerable promise in the UK military, on the Tornado and Sentinel aircraft fleets [2]. Other similar work on intermittent fault detection has been done by Muja and Lamper (2012) [75], and Smith et. al (2009) [76].

\subsubsection{Built-In-Test}

As electronic equipment evolve into ever more complex systems, they increasingly depend upon BIT to provide in-situ fault detection and isolation capabilities, particularly in low volume electronic systems in the military, aerospace and automotive sectors. BIT is a coherent assortment of on-board hardwaresoftware elements, enabling a diagnostic means to identify and locate faults as well as error checking. Its importance has therefore increased with system complexity, as it enables equipment maintainability through better testability (IEC 60706-5 [58]). In accordance to the ARINC $672^{11}$ [77], diagnostic testing should consider multiple level tests (e.g. during operation and at different maintenance echelons). Historically, it is recognized that BIT had been designed and used primarily for in-field maintenance by the end user, but they are now used in evermore diverse applications which include oceanographic systems, multichip modules, large-scale integrated circuits, power supply systems, avionics and also in passenger entertainment systems for the Boeing 767 and 777 [72]. BIT is used to indicate system status, providing valuable information to locate the exact system components (that need to be replaced) and to indicate whether or not a system has been assembled correctly.

Failures reported by BIT tests can be costly, and are likely to result in unit replacements, recertification, or inevitable loss of availability of the equipment [1]. Even though these checks may be designed as a means to detect and locate equipment faults, there are a variety of shortcomings which contribute to the NFF phenomena. Many experts advocate that the design of a BIT system is a non-trivial task and rely deeply on the knowledge of all the system interactions [5, 43]. Due to this, it is often difficult to define a fixed set of test procedures that can verify the full functionality of a component. This has led to log reports containing spurious fault detection. For example, operator/pilot reports of faults often do not always correspond to the test $\log$ s, resulting in overlooked maintenance issues. Also, even with the sophistication of modern tests, there is still a major issue of removed units, reported by the test to be at fault, but upon testing being found to have no faults, or even faults that do not correlate to the BIT reports. As well as the false alarm

\footnotetext{
11 _this has been discussed in Part 1 Section 4.
}

issue, other factors such as assessment coverage and inappropriate parameter limits, can in turn, contribute to NFF events [2].

Assessment coverage deals with the nature of the BIT (which could be designed in several different ways), making the checks dependent on the monitored equipment and system scale. A system-wide BIT will either be centralized, where dedicated hardware is used to control all functions, or decentralized, where a number of test centers can be incorporated and processed at the Line Replaceable Unit (LRU) level ${ }^{12}$. Decentralization of tests enable the ability to check the functionality of key circuits, helping to identify problems much closer to the root causes than is the case in the centralized view making for a cost-effective assembly and maintenance operations [43]. The nature of BITs will be, in some way, dependent upon a set of pre-defined statistical limits for the various parameters which are being monitored. It is important to recognisee at this point that BIT will report failures for following two reasons:

1. A specified parameter has exceeded a set threshold value

2. The noise of the BIT measurements throws the test results outside of the testing limits when the System-Under-Test (SUT) meets required specifications.

The first of these is a direct result of component failure, for example a burnt out resistor. The second occurs when a measured parameter which has noise is measured by an instrument having its own noise, this is common in integrated manufacturing processes, digital system timings and radar systems [78]. One of the areas of concern within these statistical limits is that they may have been inappropriately set without a true understanding of hardware-software interactions or the nature of the equipment's operating environment. This will therefore inevitably lead to BIT false alarms.

\subsubsection{Other Methods}

Some other techniques which have been proposed include:

1. DC resistance: Traditionally, these techniques have been utilized to monitor the reliability of electronic components, as it is well suited for identifying electrical continuity. However, these methods do not often provide any early indication of failure (of physical degradation), and may not be sensitive enough for future electronics that operate at higher frequencies.

2. RF impedance: Kwon (2010) [72] worked on developing an RF impedance method to provide an early indication of interconnect failures. The technique has better sensitivity towards degradation, as compare to its DC counterpart, due to the phenomenon known as the skin effect. The method takes advantage of the surface concentration of high speed signals (depending on the material characteristics) being passed through the connection whilst monitoring the frequency response.

\footnotetext{
${ }^{12}$ A Line-Replaceable Unit (LRU) level is the lowest level when a modular (or sub-unit) item of the system can be easily replaced and quickly interchanged.
} 
3. Functional process methodology: In order to eliminate warranty related NFF events, Izquierdo and Ceglarek (2009) [33] demonstrated a methodology based on design tolerances that integrate service (or warranty) data with manufacturing measurement, and existing product models.

\section{Improvements in Test Abilities}

Testability, as defined by IEC 60706-5 [72] is a quantitative design characteristic which determines the degree to which an item can be tested under stated conditions. As more sophistication is added to electronic systems, the ability to maintain them is becoming ever more difficult and costly. Standard testing using Automatic Test Equipment (ATE) usually includes features such as timing, signal strength, duplicating the operating environment, loading, fanout and properly interconnecting the Unit Under Test (UUT) $[60,79,80,81,82]$. The idea of ATE is to force the UUT to fail without actually injecting faults. The ability to do this is directly related to its testability. Testability is a design-related characteristic, which if designed well will provide the capabilities to confidently and efficiently identify existing faults. The number of tests and the information content of test results, along with the location and accessibility of test points, define the testability potential of the equipment. The two attributes which must be met for testability success are:

1. Confidence: this is achieved by frequent and unambiguously identifying only the failed components or parts, with no removals of good items.

2. Efficiency: this is achieved by minimizing the resources required to carry out the tests and overall maintenance action. This includes minimal yet optimized man-hours, test equipment and training.

It is evident that the conventional ATE methods used within the maintenance line as required from the testability design are not successful $[2,5,21,83]$. They perhaps are not carrying the necessary levels of confidence and efficiency, or are inappropriate, in the many industries which are suffering NFF difficulties. If testability as a design characteristic was successful, NFF would not be so problematic. This is particularly evident in the case of attempting to detect and isolate intermittent faults at the test station. The ability to test for short duration intermittency at the very moment that it re-occurs using conventional methods is so remote that it will almost certainly result in a NFF. The one major issue with designing component testability is that the focus is on functionality and integrity of the system [46].

Other difficulties with testability are that in most cases, there is a complete lack of information regarding standardized tools for the evaluation of Design for Testability (DfT) ${ }^{13}$. For testability to be consistent within the design process, to achieve the necessary levels of confidence and efficiency, these standard

\footnotetext{
${ }^{13}$ There are design techniques that are added to obtain certain testability features during hardware product design. The premise of the features is that they can make it easier to develop and apply manufacturing tests, and to validate that the product hardware contains no defects that could, otherwise, adversely affect the product's correct functioning, e.g. boundary scanning.
}

definitions, procedures and tools must be developed. A testability evaluation should not only provide predictions but also redesign information when testability attributes are predicted to be below the acceptable levels. There are three testability attributes which can be identified [84]:

1. Fraction of Faults Detected (FFD): Ideally this should be $100 \%$. Any fault not detected by either the BIT, BITE or ATE can result in total loss of the system integrity and hence functionality. In reality some faults, not safety/mission critical can be tolerated and so a FFD less than $100 \%$ may be acceptable when designing for testability.

2. Fraction of Faults Isolated (FFI): If a detected failure is not isolated quickly and efficiently with high confidence, then the system may end up being kept out of operation for significant periods of time. The result of this leads to pressure on maintenance personnel who are then likely to adopt the 'shotgun approach" ${ }^{14}$, of speculative LRU replacements adding pressure and complications to the sparing and logistics processes increasing life-cycle costs. Appropriate measures of FFI include Mean Time to Fault isolation (MTFI), Mean Time to Repair (MTTR) and rates of NFF.

3. Fraction of False Alarms (FFA) or Rate of False Alarm (RFA): This is a measure of the rate at which detected faults results as a false alarm upon investigation. It is computed as a time-normalized sum of false alarms, where the normalization is either calendar time or operating hours. High FFA will also lead to maintenance pressures and the 'shotgun' effect.

\subsection{Detecting Blind Spots}

When it is suspected that NFF occurs due to a lack of fault coverage by the ATE (or BITE), there comes the requirement to use additional tools which are capable of identifying the root cause of the problem. Ungar and Kirkland (2003) [79] argue that to achieve this, an understanding of the Physics-of-Failures $(\mathrm{PoF})^{15}$ within the operating environment is needed. Once this is known, appropriate test equipment can be selected to support the ATE which through interpretation of the physics, for example, of circuits under the test environment to be used as fault locators; a capability often beyond that of standard ATE. In fact, Kimseng et al. (1999) identified a PoF process to identify, induce and analyze not only failure mechanisms causing intermittent failures but also high warranty returns and NFF problems of the digital electronic [85]. As previously discussed, many of the faults which contribute to NFF events in electronics are of an intermittent nature. These usually provide a challenge

\footnotetext{
14-i.e. the maintainer is left to troubleshoot the system using their 'best guess', which will often result in the replacement and removal of modules that are perfectly good.

${ }^{15}$ Physics-of-Failures $(\mathrm{PoF})$ is a concept utilized to understand the processes and mechanisms that induce failure within a component. This includes studying physical, chemical, mechanical, electrical, or thermal aspects which influence the performance of the component over time, until it eventually fails to meet any system requirements.
} 
to signal processing algorithms which are often designed with permanent faults in mind [86]. Some work on resolving such issues have been carried out using algorithms that make use of Bayesian networks to decompose large systems containing multiple components that may potentially fail during operation [87]. Such probabilistic approaches often prove useful for study the performance behavior of underperforming subsystems that eventually lead to a system failure. Typical circuits are usually tested one at a time, or just a few circuits at a given time and unless the intermittent fault occurs within the time window of the test; the fault will go undetected [74]. This is compounded further by digital averaging of results, which indicates that conventional testing equipment do not provide effective test coverage for intermittency; one of the major drivers for NFF.

Other alternatives to address the intermittency problem, which try to use traditional measurements, include methods such as tracking and comparing circuits down to fractions of a milliohm, one-circuit at a time, against long running records of similar measurements. However there are some major limitations to this approach: when an intermittent circuit is in a temporary working state it will generally pass such tests and only those approaching hard-failure status will be detected this way. Also, measuring 'fractions of a milliohm' and attempting to take meaningful action based on these values is extremely difficult, time-consuming and requires precise control in the test set-up and test environment.

Appropriate test equipment is required to address the intermittency issue and to resolve all of the variables causing this unpredictability providing the maintainer with a quick and comprehensive route to a successful outcome. Overcoming the testing challenges posed by intermittent problems require a different approach to that of using conventional digital equipment predicated on accuracy of measurements and time-consuming results analysis. Truly effective and practical detection of intermittency requires improved test coverage and, consequently, vastly improved probability of detection.

There are also a variety of other high profile integrity testing methods currently being championed. Most notable of these are the use of X-ray and thermal imaging. X-ray inspections that can highlight shorts, or coupling faults buried within the layers of multiplayer printed circuit boards non-invasively. Sankaran et al. (1998) [88] discusses the use of X-ray laminogrophy for accurate measurements of solder joint structures through 3D image reconstruction using artificial neural networks. Automated inline systems based on X-ray transmission have several advantages over optical inspection. Optical inspection is restricted to surface inspection of visible solder joints. Consequently leads and ball grid arrays cannot be inspected by optical means. More sophisticated features concerning the solder volume, fillet, voids and solder thickness can reliably be determined only by X-ray transmission. Therefore, by X-ray inspection, generally a better test performance is achieved in terms of false alarm rate and escape rate and it is to be favored for closed loop process control [89].

The use of infrared imaging for non-destructive evaluation of electrical component integrity is a well-known practice [90]. The basic principle of using infrared imaging as an integrity test is that faulty connections and components in an energized circuit operating will begin to heat up before they fail, the use of a thermoscope, would scan the devices in the circuit from one end to another and the hotter the target the more energy that it will emit in the infrared portion of the electromagnetic spectrum. For many electrical components, such as resistors and capacitors the build-up of heat will be entirely normal, but for many components the build-up of heat or even lack of heat will indicate a problem.

\subsubsection{Environmental Testing}

The environmental conditions of a product (or system) can also be analyzed to assess its on-going health, and to provide an advance warning of failure [54, 91]. Products often behave differently during varying operational conditions (normal or extreme) which result in fault symptoms manifesting themselves only under those specific conditions. Examples include when temperature widely fluctuates or stress is applied in the form of vibration; conditions which will not normally be present during laboratory testing. Most products will undergo environmental testing to prove their reliability and robustness under the most extreme operating conditions as part of their certification process, but a more subtle set of environmental testing can also be used as part of the maintenance process which tries to simulate a more normal mode of operation. In effect, when designing for DfT, information-gathering exercises can be designed to study system behavior where such variation are present, i.e. Design of Experiments (DoE) [53]. These may provide essential statistical information for planning experiments on process models, in order to obtain data that can yield valid and objective conclusions.

In any case, there are three main environmental conditions which should be controlled for a good diagnostics test; humidity, vibration and temperature. However testing standards do not require these environmental factors to be done together [2]. Each of these will depend on many factors for example, temperature and humidity will fluctuate with variables such as altitude, time of year, current weather patterns whilst vibration is dependent upon such things as smoothness of roads/runways, location in the vehicle, and the vehicle activity (i.e. a fighter aircraft cruising or in a battle scenario). These three conditions can be simulated with relative ease through the use of market available environmental chambers. White and Richardson (2011) [92] provide an overview of the differing types available and the variety of tests which can be carried out in them to investigate the event of NFF issues for aircraft assemblies. In this research paper, the authors also warn that environmental testing is not the definite solution to identifying all faults. There is also a need to get operational information which includes field data, maintenance history and failure probabilities to determine if the failure in the unit is real, or if it is in a different unit or even a false alarm. However, gaining this information can be tricky and would require additional work on behalf of pilots (or operators) in recording the events which led to the failure signal along with changes to procedural practices in maintenance record keeping (or retrieval). Often an overlooked area when considering an environmental test is the orientation of the UUT 
when embedded within its operating platform. The orientation can mean that differing components are more affected by vibration than if the UUT was in a different position and so the orientation of the UUT should be a consideration when undergoing environmental testing.

\subsubsection{Tracking Spare Parts}

The ability to recognisee rogue units ${ }^{16}$ is of paramount importance in mitigating the effects of NFF events and to ensure operating safety, particularly in the case of an aircraft. The key to distinguishing a rogue unit is to implement the necessary procedures to track rogue units by serial number showing the date installed and removed, the platform on which the unit was installed, number of operating hours/cycles, number of hours since its last overhaul and a solid reason for the generated removal codes. In addition to this, the history of the operating platform (be that a wind turbine, aircraft or train) needs to be recorded with an easy to use retrieval system [2]. The importance of such historical data is to aid in determining the exact effects the failure has on the overall system and whether the replacement of the unit offers a high level of confidence of rectifying the problem.

Some airlines in the UK, operate within a spare parts pool where the policy is that if a unit is returned to the pool labeled NFF more than three times then that unit will be scrapped. This has the advantage that the spare parts pool will become less polluted with units which are rogue. However, this only encourages the culture of accepting NFF and not searching out the root cause which may be a fundamental manufacturing flaw present in equivalent units, such as a batch of faulty capacitors which have been used in the unit's production. Likewise, it could be a system design flaw leading to integration faults. Either way, scrapping units in this way will inevitably lead to an increase in costs [5].

Other airlines routinely tag and track units that come back with similar reported failure symptoms multiple times. These tagged units are then subjected to special testing that is not usually required such as thermal shock and environmental tests. Units tagged as rogue are also tracked by the tail number of the aircraft from which they came. Technicians then 'monitor and track' repetitive serial numbers using specialized tools to help determine if the unit is a repetitive problem or if the problem is fundamentally an issue with the aircraft [93]. In the case of airlines which are contracted into a spare parts pool utilized by several airlines the lack of 'tracking by design' of units suspected of being rogue means that an airline has no information regarding any unit that they take from the pool.

Advanced tracking methods have begun to gain popularity particularly in the aircraft industry which is based upon RFID tracking for predictive maintenance [94]. In the repair process, multiple operations are conducted to repair a complex engineered machine (such as an engine) which would include dismantling, inspection, repairing, maintenance and re-

\footnotetext{
${ }^{16}$ Units which have been taken out and sent back for repair multiple times are tagged as 'rogue units'.
}

assembling. Tracking and tracing of the status of these processes and operations provides critical information for decision making. This tracking and tracing is often performed manually but the adoption of RFID as an automatic identification technology has the potential to speed up processes, reduce recording errors and provide critical part history [95]). The use of RFID technology to track units within a spare parts pool providing full service histories to the current user [96] has also provided the ability to reduce the number of NFF events identifying rogue units in the spare parts pool; reducing costs attributed to phantom supply chains.

The use of RFID technology over recent years has begun to be taken very serious by major aerospace manufactures (such as Messier-Dowty) for use in future landing gear health management systems and the world's two dominant airlines Boeing and Airbus. In 2005, Boeing announced that in order to improve its ability to track and maintain service histories of its parts, it would require many suppliers of high-value parts to its new 787 Dreamliner aircraft to place RFID tags on all parts before shipping them to Boeing. Even though RFID tagging is considered an expensive option, Boeing argues that for the additional cost of $\$ 15$ per tag for a $\$ 400,000$ primary flight computer, the life-cycle information gained would more than justify the additional expenditure to their customers [97]. In early 2012, Boeing Commercial Aviation Services were still awaiting Federal Aviation Administration (FAA) certifications for RFID tracking systems aimed as a standard component on all new 737, 777 and 787 commercial aircraft as well as a variety of their military aircraft. Similarly, Airbus is also promoting the adoption of RFID in the aircraft industry, and are developing RFID part tracking systems for their new A400M military transport plane as well as for the A380 commercial jet [98].

\section{Discussion on Gaps in Literature}

In the past few decades, there has been a great deal of research in order to address the NFF issue but solutions to mitigate the problem are certainly not universal even within some individual organizations, let alone across a common industry sector. Some of this effort is being directed at the design and production stages where there is a need to create more fault-tolerant systems which perhaps incorporate in-built redundancy, or self-testing mechanisms. Also, there is a requirement for some thorough research effort into understanding intermittency. Understanding intermittent faults will rely on the ability to describe the various interactions accurately and how mechanical, software and electronic elements all have to interact together. Modeling of intermittent faults will be required, but will need to include probabilities of fault detection and the effects intermittent failures have on other dependant systems. A thorough understanding of individual systems will be required in order to provide fault models and models that deal with false BIT alarms and the root causes of BIT deficiency. In some industries and individual companies, adopting better prognostics has ensured that important operational parameters are monitored at all times to identify adverse and out of limits variations. These technologies have helped to introduce a change 
from a policy of reactive maintenance, to a predictive policy which would concentrate on providing vital information on the root causes of failures, which is not provided with traditional BIT/BITE. Other technology improvements such as the use of RFID technology has been adopted to track units within the supply chain, and to monitor the complete service history of items while they are in the supply chain. Such technology solutions will go some way to mitigating NFF but what is needed is a comprehensive approach dealing with organizational, procedural and behavioral issues as well as all the technical issues. The ability to map a NFF event from the initial reported failure through the entire maintenance process would provide invaluable information identifying the critical operations and procedures which are failing.

From the literature research within this paper, it is possible to identify the following core gaps in NFF failure related research:

1. The Problem of Intermittency: It is clear that intermittent fault occurrences are a major technical root cause of NFF and that there is a clear lack of fundamental understanding on intermittency in electronics. Also, there is clear evidence to suggest that the current technology in use for detecting and locating the source of the intermittency is inadequate. If NFF becomes worse over time despite improved management processes, then the cause is likely to be an inadequate equipment for testing electrical intermittence. In this case, there needs to be a change in the way an electronic device or wiring harness is tested in order to solve the problem. The nature of the NFF needs to be understood and tracked within equipment and if there is an intermittent NFF problem then the equipment requires NFF intermittency-capable testing equipment.

2. Integrity Testing: Most standard maintenance procedures employ only functional testing which determine if the equipment is within appropriate tolerances for service. They do not capture the level of 'damage' or 'degradation' within the equipment, information which could be vital for predicting the probability of intermittency or other failure modes. Integrity testing should be incorporated into the maintenance process and data management techniques should then be developed to provide a diagnostic history and prognostic capability. It is proposed that assessments of currently available testing methods should be investigated and developed to provide this integrity assessment capability.

3. Maintenance Manuals: The current standard in troubleshooting guidance is the Fault Isolation Manual. These manuals can be costly to produce and maintain within a dynamic environment, and are often tied to the technical publications cycle, usually meaning several months between updates. Depending on organizational and cultural factors, it might not be effective to put all the troubleshooting knowledge in a paper-based (or electronic) guidance format, and hence a diagnostic reasoning engine might be an effective system to implement [42].

4. Achieving Diagnostic Success: In order to improve diagnostic success rates, improvements need to be made to pro- cesses, procedures and technology which have failed. Initial research shows that work towards this goal is patchy and there is definitely more to do. There is almost certainly not one universal industrial solution. The current key areas for NFF mitigation are focused around understanding test coverage represented by BIT/BITE/ATE deficiencies, development of new maintenance troubleshooting tools, techniques and concepts as well as changes to management processes. Accurate fault models, fault/event trees and system understanding, are paramount to recognizing false BIT alarms (caused by such things as a sensor system synchronization). Also, new systematic tests should be identified in the product design. These tests would aim at allowing multiple testing of stressors, identifying weaknesses and flaws, and the critical contributors to failures before the product is put into service.

\section{Concluding Remarks}

An important part of any new research subject is the design and maintenance of a reference collection of relevant publications. To the best of the authors knowledge, the performed study has moved the body of scientific knowledge forward by reviewing existing literature related to NFF and pointing out core gaps where current efforts should be focused on. An attempt is made to comprehensively review academic journal literature and conference proceedings on the topic. The aim is to provide a general picture of the research areas, undertaken in past few decades, and create a database of the academic literature of journal publications on NFF concepts (and its applications) from 1990 to 2013 by classification and statistical analysis. It is evident that the NFF phenomenon has gained the most attention in the last decade. This is possibly due to increasing system complexities, reliability requirements and cost implications.

The article reported various occurrences and root causes that have resulted in NFF events. Current industrial practices were discussed whilst highlighting the importance of capturing and sharing as much information as possible to support rapid diagnostics and troubleshooting workflow. Furthermore, emphasis was placed on the importance of having feedback mechanisms to transfer maintenance event information to design engineers; who can use that information to determine how best to employ various diagnostics technologies (e.g. BIT, diagnostic reasoning, ATE, etc) to detect failures in the future. It seems that the role of having more specific standards, solely focusing upon NFF mitigation, might become much more prominent as they can promote best practice approaches within maintenance sectors. However, solutions will not reside only within different maintenance echelons, but should also focus on a much broader scope; considering factors such as design, manufacturing, testing, organizational imperatives, operator priorities, technological capabilities, contractual agreements and financial management.

This study highlights the fact that the majority of research, that has been published, primarily lies within aerospace proceedings (such as IEEE publications and other engineering out- 
lets). Surprisingly there are no dedicated textbooks on the topic, and the authors strongly feel that the maintenance community will benefit from its publication. Also, the authors advocate that the focus of published material needs shifting from the technical issues towards the business side. This could be used as a opportunity to quantify the costs involved in NFF events, and might influence the way contractual agreements are being setup now-a-days. Each industry sector approaches NFF differently i.e. OEM, maintenance suppliers and operators, manufacturer, etc. When unplanned maintenance regimes are initiated, the costs along the supply chain, warranty, downtime, operational fines are expected to raise concerns. In either case, researchers and scientists should target to publish NFF related research in management and business journals to emphasize its importance. This will help to promote knowledge, in addition to overcoming barriers in NFF investment, and the lack of a business case, due to no standardized methods (or metrics) for costing impacts.

\subsection{Future Perspectives}

The core areas where efforts should be focused on:

1. Establishing a consistent NFF taxonomy.

2. Failure Knowledge Bases, novel FMEA tools and troubleshooting guides specific for NFF to improve diagnostic success rates.

3. Development of assessment tools to assess maintenance capability (or effectiveness) which may include:

i Recording and cross referencing test station configuration and performance statistics with NFF occurrences. This includes statistics on equipment calibrations.

ii Ensuring that the testing environment is correct and investigations into whether testing procedures need modification to consider multiple environmental factors (humidity, temperature, vibration etc) simultaneously

4. Introduction of integrity testing as complimentary to standard ATE (functional) testing procedures.

i Integration of on-board health and usage monitoring.

ii Standardization for intermittent testing and procedures for dealing with intermittent fault occurrences.

5. NFF specific maintenance cost models for design justification and NFF tracking.

6. Modeling of complex interactions between system (and components) and their physics of failure.

7. Modeling of intermittent failures from a fundamental perspective including standardized testing equipment and procedures.

\section{Acknowledgements}

This research was partially supported by the Engineering and Physical Sciences Research Council (EPSRC), Ministry of Defence, BAE Systems, Bombardier Transportation and Rolls Royce. The Authors would like to express their thanks to Casebank Technologies Inc, Copernicus Technology Ltd, FlyBe UK and the RAF for sharing their experience with NFF problems.

\section{References}

[1] J. Chen, C. Roberts, P. Weston, Fault detection and diagnosis for railway track circuits using neuro-fuzzy systems, Control Engineering Practice 5 (16) (2008) 585596.

[2] C. Hockley, P. Phillips, The impact of no fault found on through-life engineering services, Journal of Quality in Maintenance Engineering 18 (2) (2012) 141-153.

[3] J.-S. Jeong, S.-D. Park, Failure analysis of video processor defined as no fault found (nff): Reproduction in system level and advanced analysis technique in ic level, Microelectronics Reliability 49 (9) (2009) 11531157.

[4] M. Pecht, R. Jaai, A prognostics and health management roadmap for information and electronics-rich systems, Microelectronics Reliability 50 (3) (2010) 317-323.

[5] P. Soderholm, A system view of the no fault found (nff) phenomenon, Reliability Engineering \& System Safety 92 (1) (2007) 1-14.

[6] I. James, D. Lumbard, I. Willis, J. Goble, Investigating no fault found in the aerospace industry, in: Reliability and Maintainability Symposium, 2003. Annual, 2003, pp. 441-446, iD: 1.

[7] V. Challa, P. Rundle, M. Pecht, Challenges in the qualification of electronic components and systems, Device and Materials Reliability, IEEE Transactions on 13 (1) (2013) 26-35.

[8] B. Sood, M. Osterman, M. Pecht, Tin whisker analysis of toyotas electronic throttle control, in: CircuitWorld, Vol. 37, 2011, pp. 4-9.

[9] T. Jin, B. Janamanchi, Q. Feng, Reliability deployment in distributed manufacturing chains via closed-loop six sigma methodology, International Journal of Production Economics 130 (1) (2011) 96-103.

[10] N. M. Vichare, M. G. Pecht, Prognostics and health management of electronics, Components and Packaging Technologies, IEEE Transactions on 29 (1) (2006) 222-229.

[11] J. K. Line, G. Krishnan, Managing and predicting intermittent failures within long life electronics, in: Aerospace Conference, 2008 IEEE, 2008, pp. $1-6$, iD: 1 .

[12] D. A. Thomas, K. Ayers, M. Pecht, The "trouble not identified" phenomenon in automotive electronics, Microelectronics reliability 42 (4) (2002) 641-651.

[13] J. H. Renner, Reliability engineering-an integrated approach at daimler chrysler, in: Integrated Reliability Workshop Final Report, 1999. IEEE International, 1999, pp. 152-153, iD: 1.

[14] H. Qi, S. Ganesan, M. Pecht, No-fault-found and intermittent failures in electronic products, Microelectronics Reliability 48 (5) (2008) 663-674.

[15] B. G. Moffat, E. Abraham, M. P. Desmulliez, D. Koltsov, A. Richardson, Failure mechanisms of legacy aircraft wiring and interconnects, Dielectrics and Electrical Insulation, IEEE Transactions on 15 (3) (2008) 808-822.

[16] G. Huby, No fault found: Aerospace survey results copernicus technology ltd (2012), Tech. rep., Copernicus Technology Ltd (2012) (2012).

[17] J. Jones, J. Hayes, Investigation of the occurrence of: no-faults-found in electronic equipment, Reliability, IEEE Transactions on 50 (3) (2001) 289-292.

[18] I. J. James, Learning the lessons from in-service rejection, in: Systems Reliability and Maintainability (Ref. No. 1999/189), IEE Seminar, 1999, pp. 6/1-6/4, iD: 1 .

[19] A. W. Gibson, S. Choi, T. R. Bieler, K. N. Subramanian, Environmental concerns and materials issues in manufactured solder joints, in: Electronics and the Environment, 1997. ISEE-1997., Proceedings of the 1997 IEEE International Symposium on, 1997, pp. 246-251, iD: 1.

[20] J. Swingler, The automotive connector: The influence of powering and lubricating a fretting contact interface, Proceedings of the Institution of Mechanical Engineers, Part D: Journal of Automobile Engineering 214 (6) (2000) 615-623.

[21] S. Khan, P. Phillips, Tackling no fault found in maintenance engineering, in: 1st Annual Symposium in No Fault Found, 2013.

[22] W. Shawlee, D. Humphrey, Aging avionics-what causes it and how to respond, Components and Packaging Technologies, IEEE Transactions on 24 (4) (2001) 739-740.

[23] S. Khan, P. Phillips, C. Hockley, I. Jennions, Towards standardisation of no-fault found taxonomy, in: 1st International Through-life Engineering Services Conference, 2012, 2012, pp. 246-253.

[24] L. Warrington, J. A. Jones, N. Davis, Modelling of maintenance, within 
discrete event simulation, in: Reliability and Maintainability Symposium, 2002. Proceedings. Annual, IEEE, 2002, pp. 260-265.

[25] G. Ramohalli, The honeywell on-board diagnostic and maintenance system for the boeing 777, in: Digital Avionics Systems Conference, 1992. Proceedings., IEEE/AIAA 11th, IEEE, 1992, pp. 485-490.

[26] I. Beniaminy, D. Joseph, Reducing the "no fault found" problem: Contributions from expert-system methods, in: Aerospace Conference Proceedings, 2002. IEEE, Vol. 6, 2002, pp. 6-2971-6-2973 vol.6, iD: 1.

[27] J. Xie, M. Pecht, Applications of in-situ health monitoring and prognostic sensors, in: The 9th Pan Pacific microelectronics Symposium Exhibits and Conference, 2004, p. 1012.

[28] I. E. Commission, IEC 60812: Analysis techniques for system reliabilityProcedure for failure mode and effects analysis (FMEA), 2006.

[29] R. Wright, L. Kirkland, Nano-scaled electrical sensor devices for integrated circuit diagnostics, Vol. 6, IEEE Aerospace Conference, 2003, p. 25492555.

[30] L. Mariani, F. Pastore, M. Pezz, Dynamic analysis for diagnosing integration faults, Software Engineering, IEEE Transactions on 37 (4) (2011) 486-508.

[31] N. G. Leveson, Role of software in spacecraft accidents, Journal of Spacecraft and Rockets 41 (4) (2004) 564-575.

[32] A. Brombacher, E. Hopma, A. Ittoo, Y. Lu, I. Luyk, L. Maruster, J. Ribeiro, T. Weijters, H. Wortmann, Improving product quality and reliability with customer experience data, Quality and Reliability Engineering International 28 (8) (2012) 873-886.

[33] L. E. Izquierdo, D. Ceglarek, Functional process adjustments to reduce no-fault-found product failures in service caused by in-tolerance faults, CIRP Annals-Manufacturing Technology 58 (1) (2009) 37-40.

[34] R. J. Meseroll, C. J. Kirkos, R. A. Shannon, Data mining navy flight and maintenance data to affect repair, in: Autotestcon, 2007 IEEE, 2007, pp. 476-481, iD: 1 .

[35] A. K. Jardine, D. Lin, D. Banjevic, A review on machinery diagnostics and prognostics implementing condition-based maintenance, Mechanical systems and signal processing 20 (7) (2006) 1483-1510.

[36] R. M. Knotts, Civil aircraft maintenance and support fault diagnosis from a business perspective, Journal of quality in maintenance engineering 5 (4) (1999) 335-348.

[37] R. Granstrom, P. Soderholm, Condition monitoring of railway wheels and no fault found problems, International Journal of COMADEM 12 (2) (2009) 46-53.

[38] S. Henning, R. Paasch, Designing mechanical systems for optimum diagnosability, Research in Engineering Design 21 (2) (2010) 113-122.

[39] P. Phillips, D. Diston, A knowledge driven approach to aerospace condition monitoring, Knowledge-Based Systems 24 (6) (2011) 915-927.

[40] F. S. Nowlan, H. F. Heap, Reliability-centered maintenance, UNITED AIR LINES INC SAN FRANCISCO CA, 1978.

[41] J. Moubray, Reliability-centered maintenance, Industrial Press Inc., 2001.

[42] P. D'Eon, Reducing nffs through knowledge sharing, in: 1st Annual Symposium on Tackling 'No Fault Found' in Maintenance Engineering, 2013.

[43] M. Pecht, Prognostics and health monitoring of electronics, John Wiley \& Sons, 2008

[44] M. S. G. . T. Force, Maintenance Program Development Document MSG3, Washington DC: Air Transport Association (ATA) of America, 1993.

[45] A. Ahmadi, P. Sderholm, U. Kumar, On aircraft scheduled maintenance program development, Journal of Quality in Maintenance Engineering 16 (3) (2010) 229-255.

[46] G. Huby, J. Cockram, The system integrity approach to reducing the cost impact of no fault found and intermittent faults, in: UK RAeS Airworthiness and Maintenance Conference, 2010.

[47] S. Kumar, N. M. Vichare, E. Dolev, M. Pecht, A health indicator method for degradation detection of electronic products, Microelectronics Reliability 52 (2) (2012) 439-445.

[48] A. H. . M. .Rausand, System reliability theory: models and statistical methods, Chapter 3, Wiley, 2009.

[49] F. H. Born, R. A. Boenning, Marginal checking - a technique to detect incipient failures, in: IEEE Proceedings of the National Aerospace and Electronics Conference, Vol. 4, 1989, pp. 1880-1886, cited By (since 1996):2.

[50] D. J. Burns, K. D. Cluff, K. Karimi, D. W. Hrehov, A novel power quality monitor for commercial airplanes, in: Conference Record - IEEE Instrumentation and Measurement Technology Conference, Vol. 2, 2002, pp.
1649-1653.

[51] S. Mathew, D. Das, M. Osterman, M. Pecht, R. Ferebee, Prognostics assessment of aluminum support structure on a printed circuit board, Journal of electronic packaging 128 (4) (2006) 339.

[52] V. Shetty, D. Das, M. Pecht, D. Hiemstra, S. Martin, Remaining life assessment of shuttle remote manipulator system end effector, in: Proceedings of the 22nd Space Simulation Conference, 2002, p. 2123.

[53] P. Lall, M. Hande, C. Bhat, J. Suhling, J. Lee, Prognostic health monitoring $(\mathrm{phm})$ for prior-damage assessment in electronics equipment under thermo-mechanical loads, in: IEEE Electronic Components and Technology Conference, 2007, p. 10971111.

[54] N. Vichare, P. Rodgers, V. Eveloy, M. Pecht, Environment and usage monitoring of electronic products for health assessment and product design, International Journal of Quality Technology and Quantitative Management 4 (2) (2007) 235-250.

[55] V. A. Skormin, V. I. Gorodetski, L. J. Popyack, Data mining technology for failure prognostic of avionics, Aerospace and Electronic Systems, IEEE Transactions on 38 (2) (2002) 388-403.

[56] R. Karim, O. Candell, P. Soderholm, E-maintenance and information logistics: aspects of content format, Journal of Quality in Maintenance Engineering 15 (3) (2009) 308-324.

[57] P.-O. Larsson-Kraik, Managing avalanches using costbenefitrisk analysis, Proceedings of the Institution of Mechanical Engineers, Part F: Journal of Rail and Rapid Transit 226 (6) (2012) 641-649.

[58] D. H. Stamatis, Failure mode and effect analysis: FMEA from theory to execution, Asq Press, 2003.

[59] C. S. Byington, P. Kalgren, B. K. Dunkin, B. P. Donovan, Advanced diagnostic/prognostic reasoning and evidence transformation techniques for improved avionics maintenance, in: Aerospace Conference, 2004. Proceedings. 2004 IEEE, Vol. 5, IEEE, 2004.

[60] L. Y. Ungar, Testability design prevents harm, IEEE Aerospace and Electronic Systems Magazine 25 (3) (2010) 35-43, cited By (since 1996):4.

[61] N. M. Morris, W. B. Rouse, Review and evaluation of empirical research in troubleshooting, Human Factors: The Journal of the Human Factors and Ergonomics Society 27 (5) (1985) 503-530.

[62] P. D'eon, M. Langley, A. Atamer, Case-based reasoning system and method having fault isolation manual trigger cases u.s. patent application 11734,862 (2007).

[63] R. C. Millar, T. Mazzuchi, S. Sarkani, Application of non-parametric statistical methods to reliability database analysis, SAE Technical Papers.

[64] A. Atamer, Comparison of fmea and field-experience for a turbofan engine with application to case-based reasoning, in: IEEE Aerospace Conference Proceedings, Vol. 5, 2004, pp. 3354-3360, cited By (since 1996):2.

[65] C. R. Sharma, C. Furse, R. R. Harrison, Low-power stdr cmos sensor for locating faults in aging aircraft wiring, Sensors Journal, IEEE 7 (1) (2007) 43-50.

[66] C. Lo, C. Furse, Noise-domain reflectometry for locating wiring faults, Electromagnetic Compatibility, IEEE Transactions on 47 (1) (2005) 97104.

[67] Y. C. Chung, C. Furse, J. Pruitt, Application of phase detection frequency domain reflectometry for locating faults in an f-18 flight control harness, Electromagnetic Compatibility, IEEE Transactions on 47 (2) (2005) 327334.

[68] C. Furse, Y. C. Chung, C. Lo, P. Pendayala, A critical comparison of reflectometry methods for location of wiring faults, Smart Structures and Systems 2 (1) (2006) 25-46.

[69] C. R. Parkey, C. Hughes, M. Caulfield, M. P. Masquelier, A method of combining intermittent arc fault technologies, in: AUTOTESTCON (Proceedings), 2012, pp. 244-249, cited By (since 1996): 1 .

[70] P. A. Smith, D. V. Campbell, A practical implementation of bics for safety-critical applications, in: Defect Based Testing, 2000. Proceedings. 2000 IEEE International Workshop on, IEEE, 2000, pp. 51-56.

[71] A. Bhatia, J. P. Hofmeister, J. Judkins, D. Goodman, Advanced testing and prognostics of ball grid array components with a stand-alone monitor ic, Instrumentation \& Measurement Magazine, IEEE 13 (4) (2010) 4247, iD: 1.

[72] D. Kwon, Detection of interconnect failure precursors using RF impedance analysis, $\mathrm{PhD}$ Thesis, University of Maryland, 2010.

[73] B. Steadman, F. Berghout, N. Olsen, B. Sorensen, Intermittent fault detection and isolation system, in: AUTOTESTCON, 2008 IEEE, IEEE, 2008, 
pp. 37-40.

[74] B. Sorensen, Apparatus for testing multiple conductor wiring and terminations for electronic systems, u.s. patent no. 8,103,475 (2012) (2012)

[75] O. Muja, D. Lamper, Automated fault isolation of intermittent wiring/conductive path systems inside weapons replaceable assemblies, SAE International Journal of Aerospace 5 (2) (2012) 579-589.

[76] P. Smith, P. Kuhn, C. Furse, Intermittent fault location on live electrical wiring systems, SAE International Journal of Aerospace 1 (1) (2009) 1101-1106, cited By (since 1996):3.

[77] A. W. 672, Guidelines for The Reduction of No Fault Found (NFF), ARINC, 2008.

[78] D. Rosenthal, B. C. Wadell, Predicting and eliminating built-in test false alarms, Reliability, IEEE Transactions on 39 (4) (1990) 500-505.

[79] L. Y. Ungar, L. V. Kirkland, Unraveling the cannot duplicate and retest ok problems by utilizing physics in testing and diagnoses, in: AUTOTESTCON (Proceedings), 2008, pp. 550-555, cited By (since 1996):1.

[80] C. Metra, S. D. Francescantonio, T. Mak, Clock faults' impact on manufacturing testing and their possible detection through on-line testing, in: Test Conference, 2002. Proceedings. International, IEEE, 2002, pp. 100109.

[81] P. O'Connor, Testing for reliability, Quality and Reliability Engineering International 19 (1) (2003) 73-84.

[82] H. Qingchuan, C. Wenhua, P. Jun, Q. Ping, Improved step stress accelerated life testing method for electronic product, Microelectronics Reliability 52 (11) (2012) 2773-2780.

[83] J. W. Sheppard, W. R. Simpson, Applying testability analysis for integrated diagnostics, Design \& Test of Computers, IEEE 9 (3) (1992) 6578.

[84] W. Simpson, B. Kelly, A. Gilreath, Predictors of organizational-level testability attributes, in: Publicatoin 1511-02-2-4179, Annapolis, Maryland: ARINC Research Corporation, 1986

[85] K. Kimseng, M. Hoit, N. Tiwari, M. Pecht, Physics-of-failure assessment of a cruise control module, Microelectronics Reliability 39 (10) (1999) 1423-1444.

[86] D. Guanqian, Q. Jing, L. Guanjun, L. Kehong, A stochastic automaton approach to discriminate intermittent from permanent faults, Proceedings of the Institution of Mechanical Engineers, Part G: Journal of Aerospace Engineering.

[87] R. Abreu, P. Zoeteweij, R. Golsteijn, A. J. V. Gemund, A practical evaluation of spectrum-based fault localization, Journal of Systems and Software 82 (11) (2009) 1780-1792.

[88] V. Sankaran, A. R. Kalukin, R. P. Kraft, Improvements to X-ray laminography for automated inspection of solder joints, Components, Packaging, and Manufacturing Technology, Part C, IEEE Transactions on 21 (2) (1998) 148-154.

[89] C. Neubauer, Intelligent $\mathrm{x}$-ray inspection for quality control of solder joints, Components, Packaging, and Manufacturing Technology, Part C, IEEE Transactions on 20 (2) (1997) 111-120.

[90] X. Maldague, Theory and practice of infrared technology for nondestructive testing, Wiley Series in Microwave and Optical Engineering, 2001.

[91] G. Deng, J. Qiu, G. Liu, K. Lv, A novel fault diagnosis approach based on environmental stress level evaluation, Proceedings of the Institution of Mechanical Engineers, Part G: Journal of Aerospace Engineering 227 (5) (2013) 816-826.

[92] R. White, B. Richardson, Anecdotal experiences on the value of limited environmental testing for the analysis of "no fault" found assemblies, in: AUTOTESTCON (Proceedings), 2011, pp. 292-296.

[93] J. Ramsey, Special Report: Avoiding NFF, Avionics Magazine, 2005.

[94] Y. S. Chang, C. H. Oh, Y. S. Whang, J. J. Lee, J. A. Kwon, M. S. Kang, J. S. Park, Y. Ung, Development of rfid enabled aircraft maintenance system, in: Industrial Informatics, 2006 IEEE International Conference on, IEEE, 2006, pp. 224-229.

[95] W. He, C. Xu, Y. Ao, X. Xiao, E. W. Lee, E. L. Tan, Rfid enabled handheld solution for aerospace mro operations track and trace, in: Emerging Technologies \& Factory Automation (ETFA), 2011 IEEE 16th Conference on, IEEE, 2011, pp. 1-8.

[96] A. Narsing, Rfid and supply chain management: an assessment of its economic, technical, and productive viability in global operations, Journal of Applied Business Research (JABR) 21 (2) (2011) 1-6.

[97] M. O'Connor, Boeing wants dreamliner parts tagged, RFID Journal (2005). 
2013-12-01

No Fault Found events in maintenance engineering Part 2: Root causes, technical developments and future research

Khan, Samir

Elsevier

Samir Khan, Paul Phillips, Chris Hockley, Ian Jennions, No Fault Found events in maintenance engineering Part 2: Root causes, technical developments and future research, Reliability

Engineering \& System Safety, Volume 123, March 2014, Pages 196-208

http://dx.doi.org/10.1016/j.ress.2013.10.013

Downloaded from Cranfield Library Services E-Repository 